Jürgen Oechsler

\title{
Vertragliche Schuldverhältnisse
}

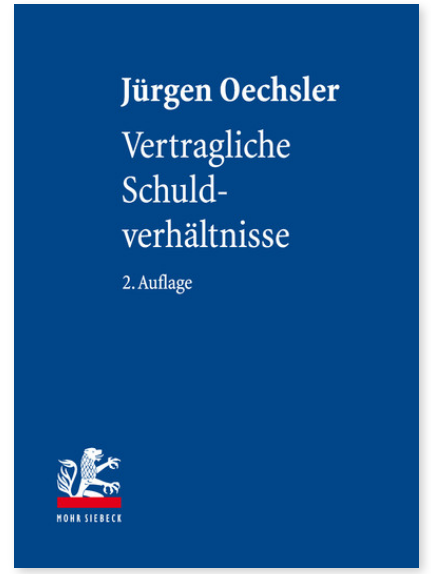

2., überarbeitete Auflage; 2017. XLI, 1124 Seiten. LbPrivR

ISBN 978-3-16-155469-8 eBook PDF 149,00€

ISBN 978-3-16-155468-1 Leinen $149,00 €$
Jürgen Oechsler bietet eine umfassende Darstellung des deutschen Vertragsrechts und seiner Prägung durch das Europarecht.

In der Neuauflage berücksichtigt Jürgen Oechsler in den Kapiteln zum »Kaufvertrag« und »Werkvertrag« das vom Bundestag im März 2017 beschlossene und am 1.1.2018 in Kraft tretende Gesetz zur Reform des Bauvertragsrechts und zur Änderung der kaufrechtlichen Mängelhaftung. Ferner wurde in das Kapitel »Reisevertrag« der Regierungsentwurf zur Umsetzung der Zweiten Pauschalreiserichtlinie (EU) 2015/2302 eingearbeitet. Im Kapitel »Geschäftsbesorgungsvertrag« wurde auch der Regierungsentwurf vom 8.2.2017 zur Umsetzung der Zahlungsdiensterichtlinie 2 (EU) 2015/2366 berücksichtigt.

"Zusammenfassend hat Oechsler ein Werk vorgelegt, bei dem es ihm erfolgreich gelingt, eine verständliche, gut lesbare und übergreifende Zusammenhänge vermittelnde Darstellung mit einer bemerkenswerten rechtlichen Präzision sowie Detailfülle zu verbinden.«

Markus Rehberg AcP 2015, 756-760

»Das sehr interessante Werk bietet in dieser 'extended version' eine ungemein lesenswerte und anregende Darstellung zum Vertragsrecht mit einer überzeugenden Schwerpunktsetzung, sowohl für das Studium als auch für die Praxis.« www.juralit.com/2013

»ürgen Oechsler [...] ist mit dem vorliegenden Lehrbuch zu den vertraglichen Schuldverhältnissen in jeder Hinsicht ein großer Wurf gelungen. Mehr als 1000 Seiten umfasst das Werk, aber langweilig wird es an keiner Stelle.« Florian Hoffmann www.kuselit.de

»An einer gründlichen Darstellung des vertraglichen Schuldrechts und insbesondere der Ab- und Herleitung der rechtspositiven Aussagen vor dem jeweiligen rechtshistorischen und -politischen Hintergrund interessierten Studenten, Referendaren und sonstigen Interessierten sei das Werk nachdrücklich ans Herz gelegt.«

Florian Kienle Die Justiz 2014, 150

»Der Horizont wird erweitert, die Argumentationskultur vertieft. Eine echte Empfehlung.» Marc-Philippe Weller JuristenZeitung 2014, 790

Für Bibliotheken gelten bei diesem Titel abweichende Konditionen; bitte wenden Sie sich an den Vertrieb.

Jürgen Oechsler Geboren 1963; Studium der Rechtswissenschaft und Orientalistik, Ausbildung und berufliche Tätigkeit in Saarbrücken, Kairo, Potsdam; Professor für Bürgerliches Recht, Handelsrecht, Deutsches und Europäisches Wirtschaftsrecht an der Johannes Gutenberg-Universität Mainz.

https://orcid.org/0000-0002-9213-6794

Jetzt bestellen:

https://mohrsiebeck.com/buch/vertragliche-schuldverhaeltnisse-9783161554698?no_cache=1

order@mohrsiebeck.com

Telefon: +49 (0)7071-923-17

Telefax: +49 (0)7071-51104 